\title{
Radical Trapping by Gold Chlorides Forming Organogold Intermediates.
}

Carmela Aprile, Mercedes Boronat, Belén Ferrer, Avelino Corma*, Hermenegildo García*.

\section{SUPPORTING INFORMATION}

\section{EXPERIMENTAL SECTION.}

UV-Vis absorption spectra were recorded with a Perkin Elmer $\lambda 35$ spectrophotometer. Laser flash photolysis experiments were carried out in a Luzchem ns laser flash system using the fourth (266 nm) harmonic of a Q-switched Nd:YAG laser for excitation (pulse $\leq 10 \mathrm{~ns}$ ) and a $175 \mathrm{~W}$ ceramic Xenon Fiberoptic Lightsource, Cermax, perpendicular to the laser beam, as a probing light. The signal from the monochromator/photomultiplier detection system was captured by a Tektronix TDS 3032B digitizer. Laser system and digitizer are connected to a PC computer via GPIB and serial interfaces that controlled all the experimental parameters and provided suitable processing and data storage capabilities. The software package has been developed in the LabVIEW environment from National Instruments and compiled as a stand-alone application. The samples contained on a Suprasil quartz $0.7 \mathrm{x} 0.7$ cuvette capped with septa were purged with $\mathrm{N}_{2}$ flow at least 15 min. before laser experiments. ${ }^{1} \mathrm{H}$ and ${ }^{13} \mathrm{C}$ NMR were recorded using a Varian Gemini 3000 (300 $\mathrm{MHz})$.

Photolysis of dibenzyl ketone or benzoyl peroxide in the presence of gold halides was carried out directly in NMR tubes dissolving the gold salts in deuterated acetonitrile and subsequently adding the radicals initiators. All the solutions were purged whit nitrogen for $15 \mathrm{~min}$. and then irradiated using water refrigerated medium pressure $\mathrm{Hg}$ lamp through quartz.

Purification of the dibenzylgold compound after photolysis in deuterated acetonitrile was performed by removing the solvent at room temperature and partitioning the residual solid between deuterated water $\left(\mathrm{D}_{2} \mathrm{O}\right)$ and deuterated chloroform $\left(\mathrm{CD}_{3} \mathrm{Cl}\right)$. The organogold compound becomes dissolved in the aqueous phase while the diphenylethane dissolves in the organic phase. The water was evaporated at $0{ }^{\circ} \mathrm{C}$ under vacuum and the organogold compound was redissolved in $\mathrm{CD}_{3} \mathrm{CN}$. ${ }^{1} \mathrm{H}$ NMR spectrum of the pure organogold compound are shown in the inset of figure S1.

Calculations were carried out using the B3LYP method with a LANL2DZ effective core potential basis set for $\mathrm{Au}$, and the standard 6-31 $\mathrm{G}(\mathrm{d}, \mathrm{p})$ basis set for $\mathrm{Cl}, \mathrm{C}$ and $\mathrm{H}$ atoms. All calculations were performed using Gaussian 03. 


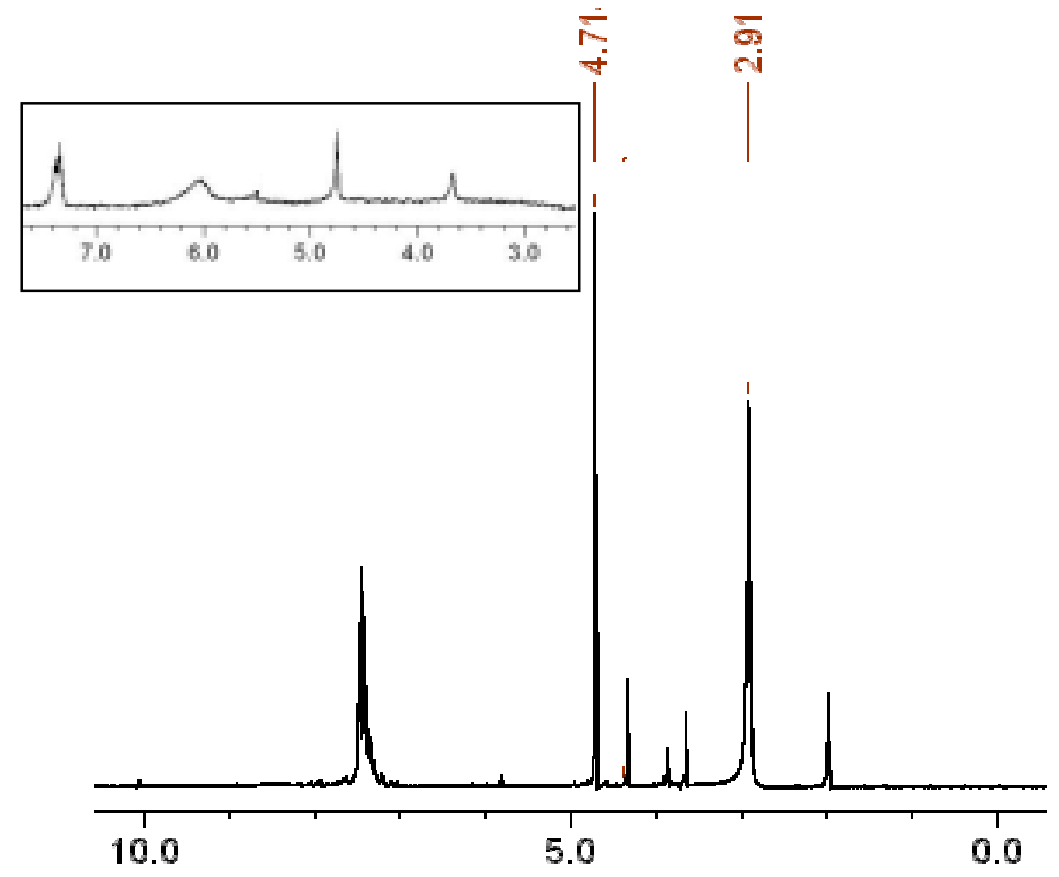

Figure S1. ${ }^{1} \mathrm{H}$ NMR spectrum recorded after $5 \mathrm{~h}$ irradiation time of a solution of dibenzyl ketone $\left(3.0 \times 10^{-2} \mathrm{M}\right)$ and $\mathrm{AuCl}_{3}\left(3.2 \times 10^{-2} \mathrm{M}\right)$ in presence of $\mathrm{CD}_{3} \mathrm{CN}$ as solvent. The peak at $4.7 \mathrm{ppm}$ corresponds to the organogold compound. The inset shows the ${ }^{1} \mathrm{H}$ NMR spectrum of the pure dibenzylchlorohydroxy aurate (III). 
Irradiation of benzoyl peroxide with $\mathrm{AuCl}_{3}$.

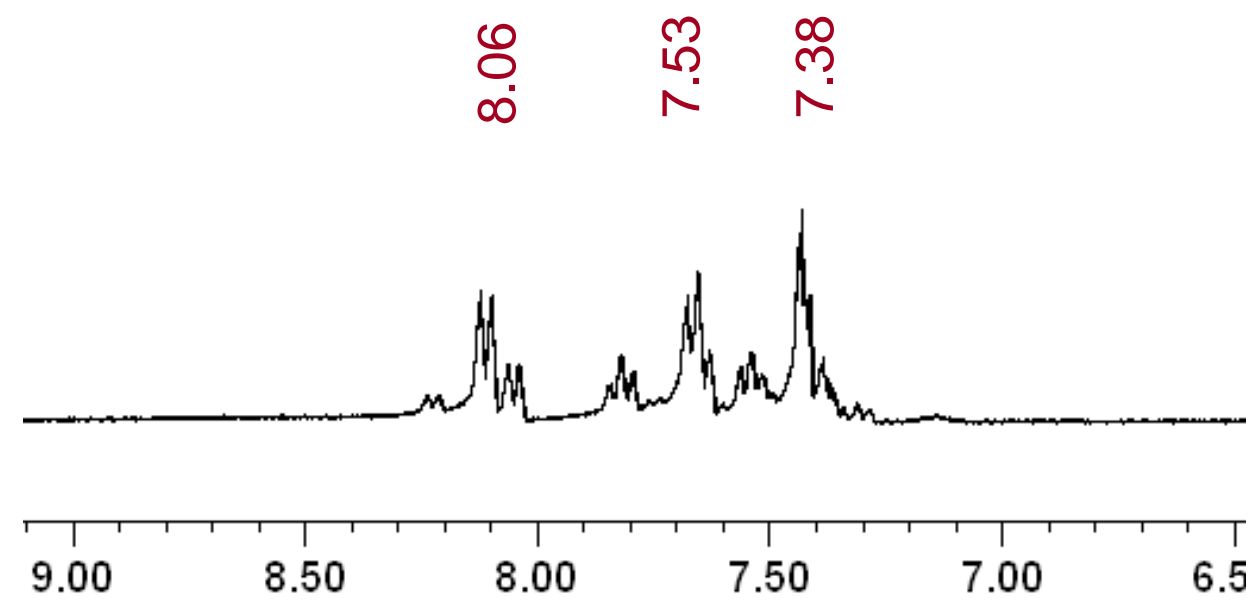

Figure S2. ${ }^{1}$ H NMR spectra of the reaction mixtures upon photolysis of benzoyl peroxide in the presence of gold halides showed a set of signals (7.38 and $7.53 \mathrm{ppm}$ triplets and $8.06 \mathrm{ppm}$ doublet), that were absent in blank controls in absence of gold chlorides.

${ }^{13} \mathrm{C}$ NMR spectroscopy also indicates the appearance of a new product when the photolysis is carried out in the presence of gold chlorides (129.8, 130.6, 131.2 and $136.0 \mathrm{ppm})$. MS shows a peak at $\mathrm{m} / \mathrm{z} 232$ that confirms the presence of $(\mathrm{Ph})_{3} \mathrm{AuCl}\left(\mathrm{M}+\mathrm{H}^{+} 464\right)$. All these spectroscopic data are again compatible with the formation of $\left[\mathrm{Ph}_{3} \mathrm{AuCl}\right] \mathrm{H}$ as indicated in scheme $1(n=3)$ arising from the phenyl radical trapping by gold halides. 


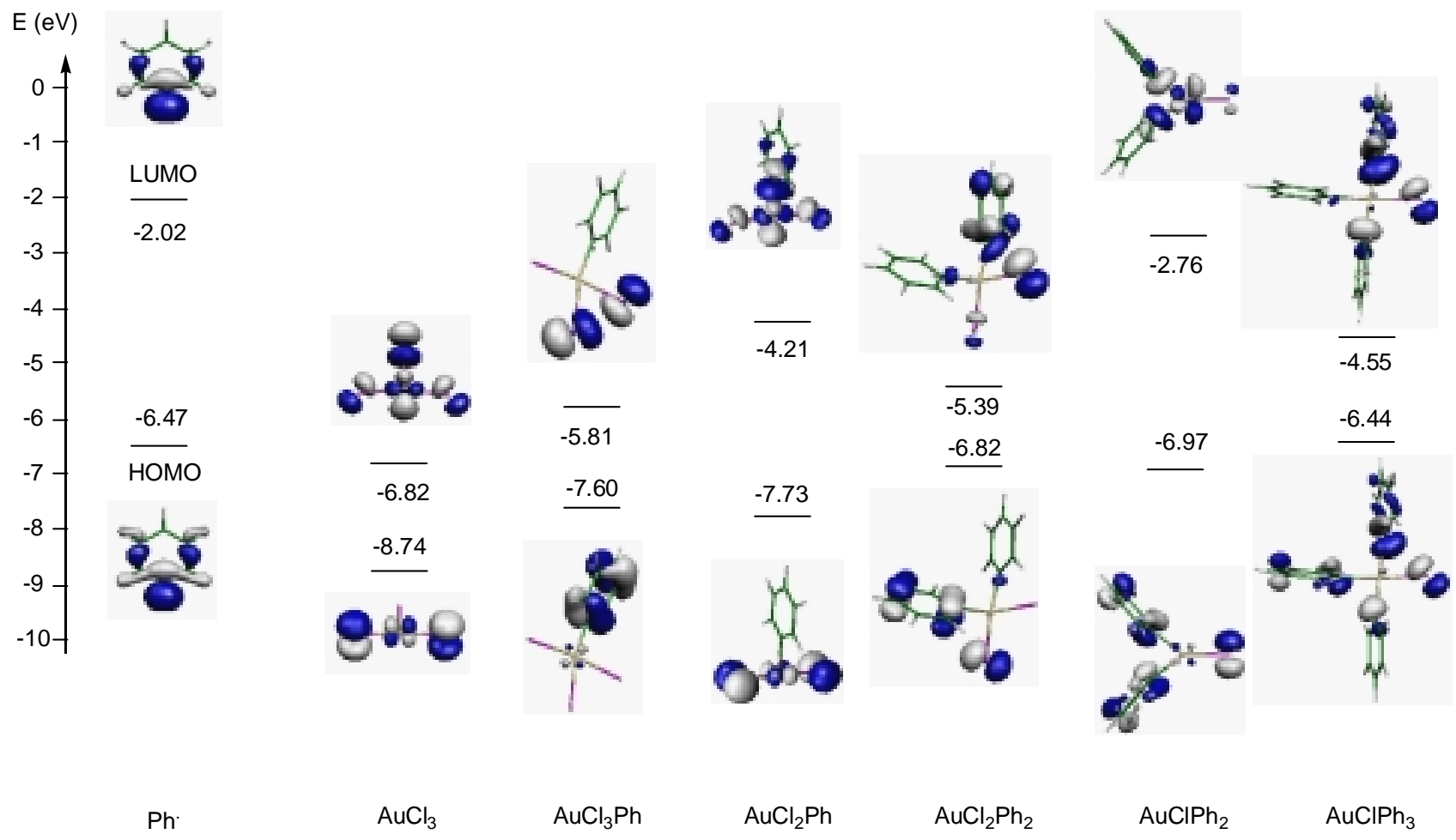

Figure S3. Diagram of energy levels and electron density of the HOMO and LUMO calculated for the species arising from the attack of phenyl radicals to $\mathrm{AuCl}_{3}$. 


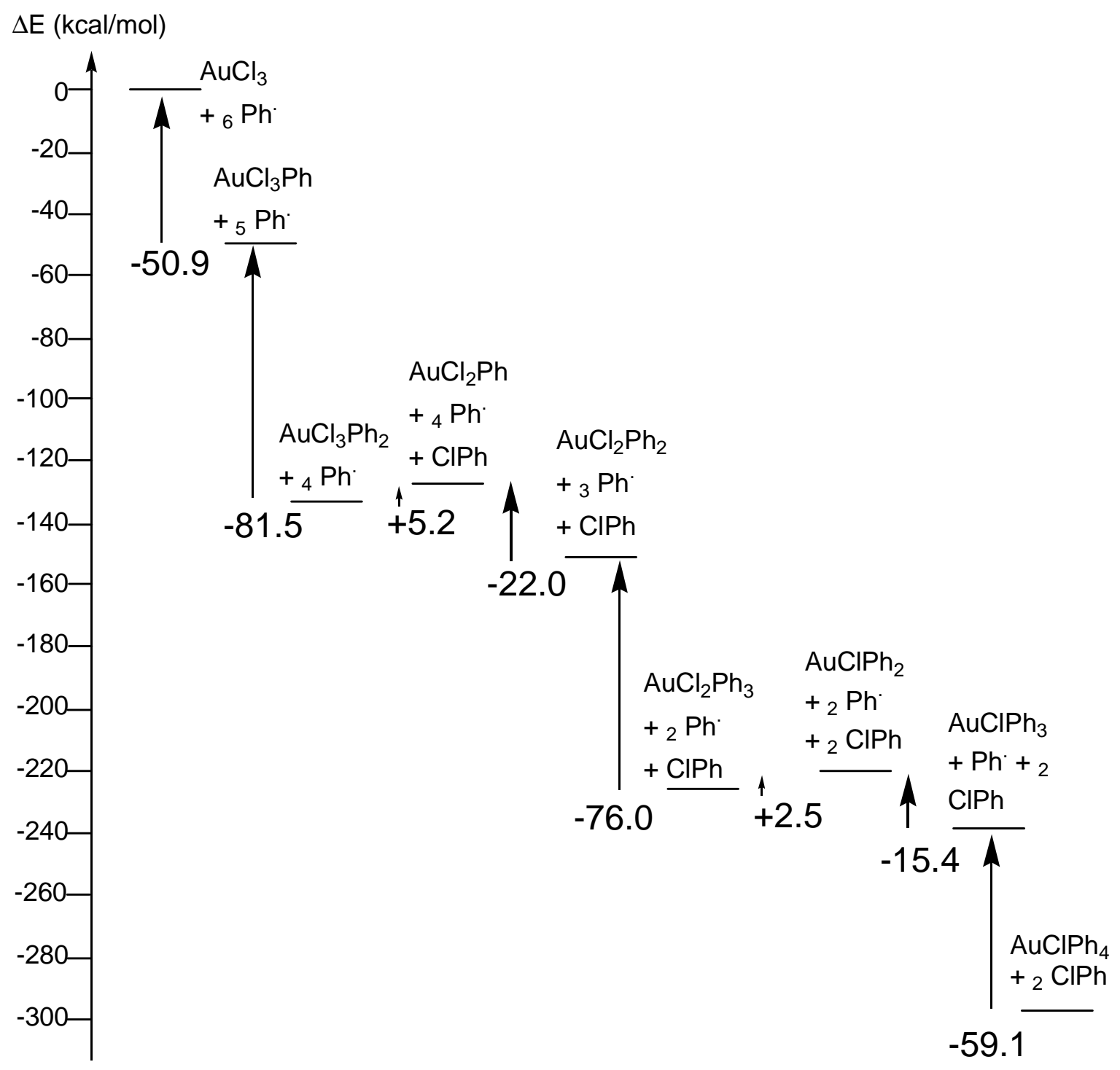

Figure S4. Diagram of the calculated energies for all the elementary steps of the proposed mechanism. 


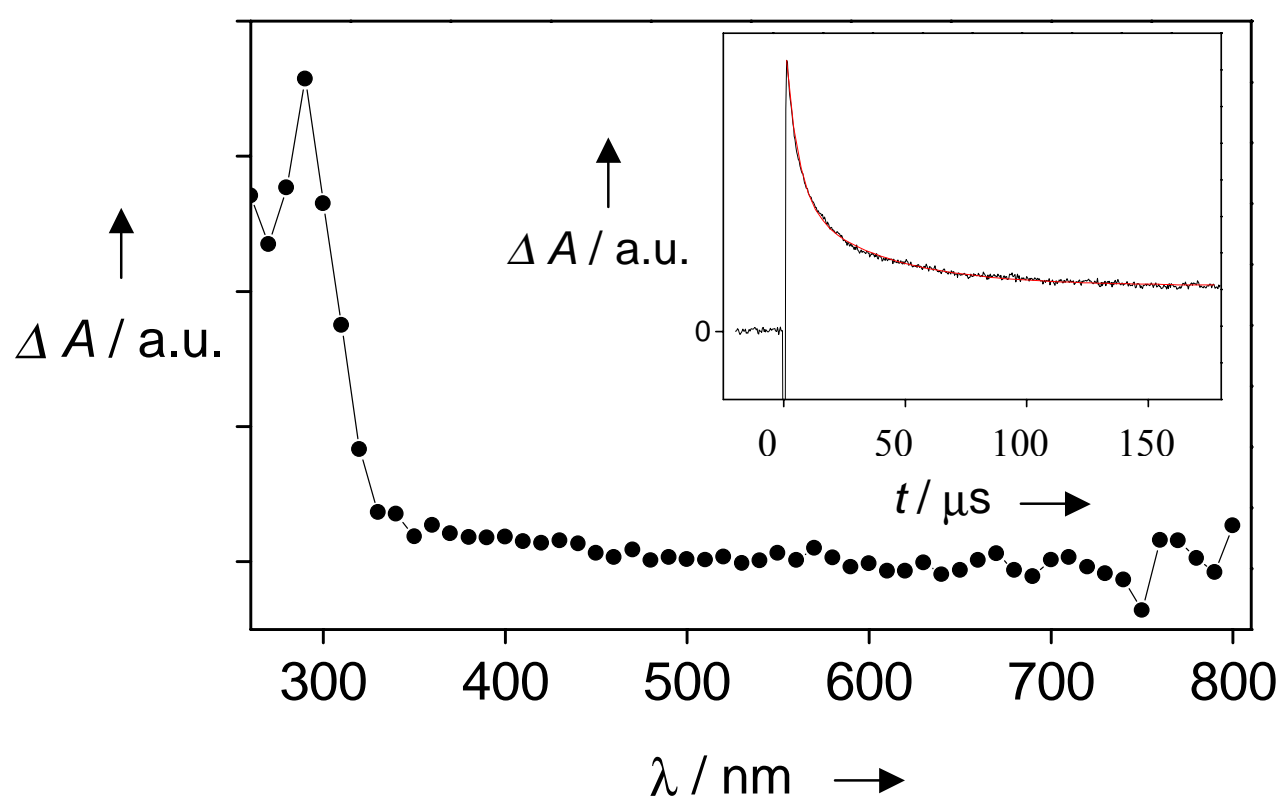

Figure S5. UV-Vis transient absorption spectrum of an acetonitrile solution of 1,3diphenylacetone $\left(1.1 \times 10^{-3} \mathrm{M}\right)$ recorded $4.8 \mu$ s after $266 \mathrm{~nm}$ laser excitation under $\mathrm{N}_{2}$ atmosphere. The inset shows the decay monitored at $290 \mathrm{~nm}$ and the best curve fit from which the lifetimes were obtained. 


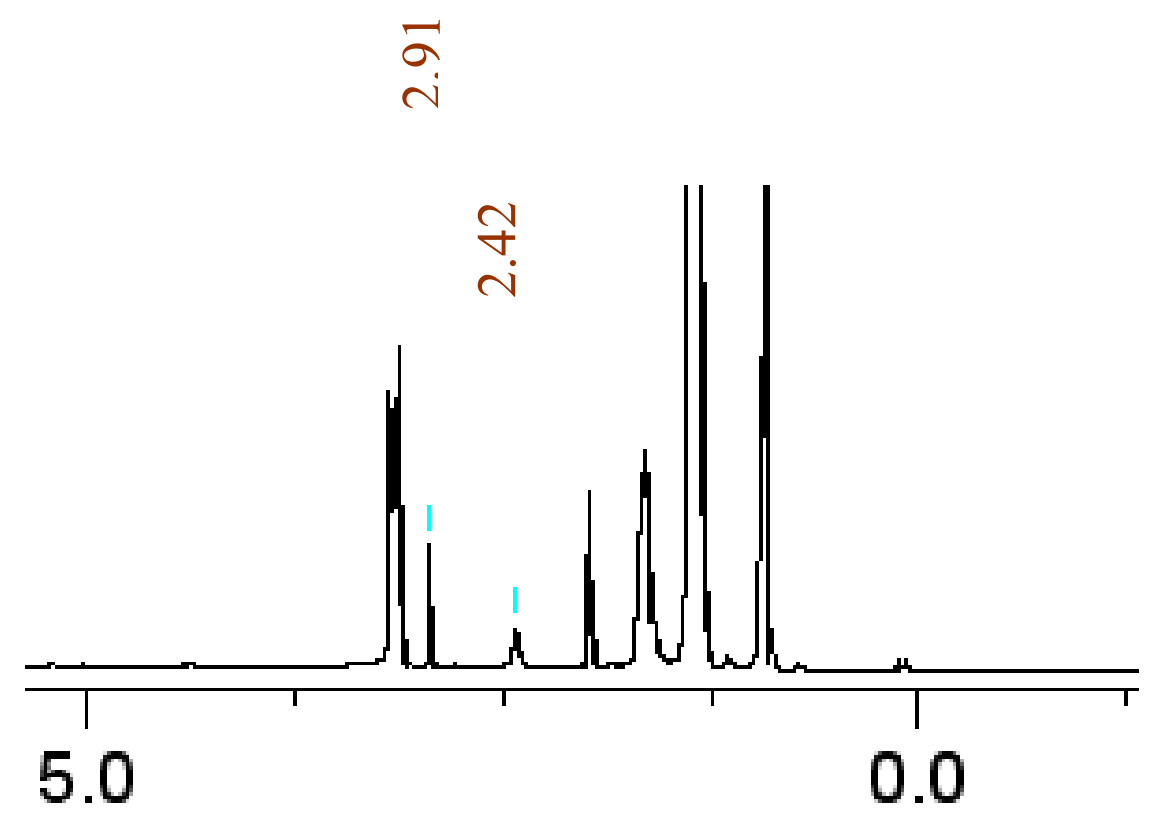

Figure S6. ${ }^{1} \mathrm{H}$ NMR spectrum recorded after 5 h irradiation time of a solution of dibenzyl ketone $\left(3.0 \times 10^{-2} \mathrm{M}\right)$ and Gold colloid in presence of $\mathrm{CD}_{3} \mathrm{CN}$ as solvent. The peak at $2.42 \mathrm{ppm}$ has been attributed to the surface-bound benzyl radical. 


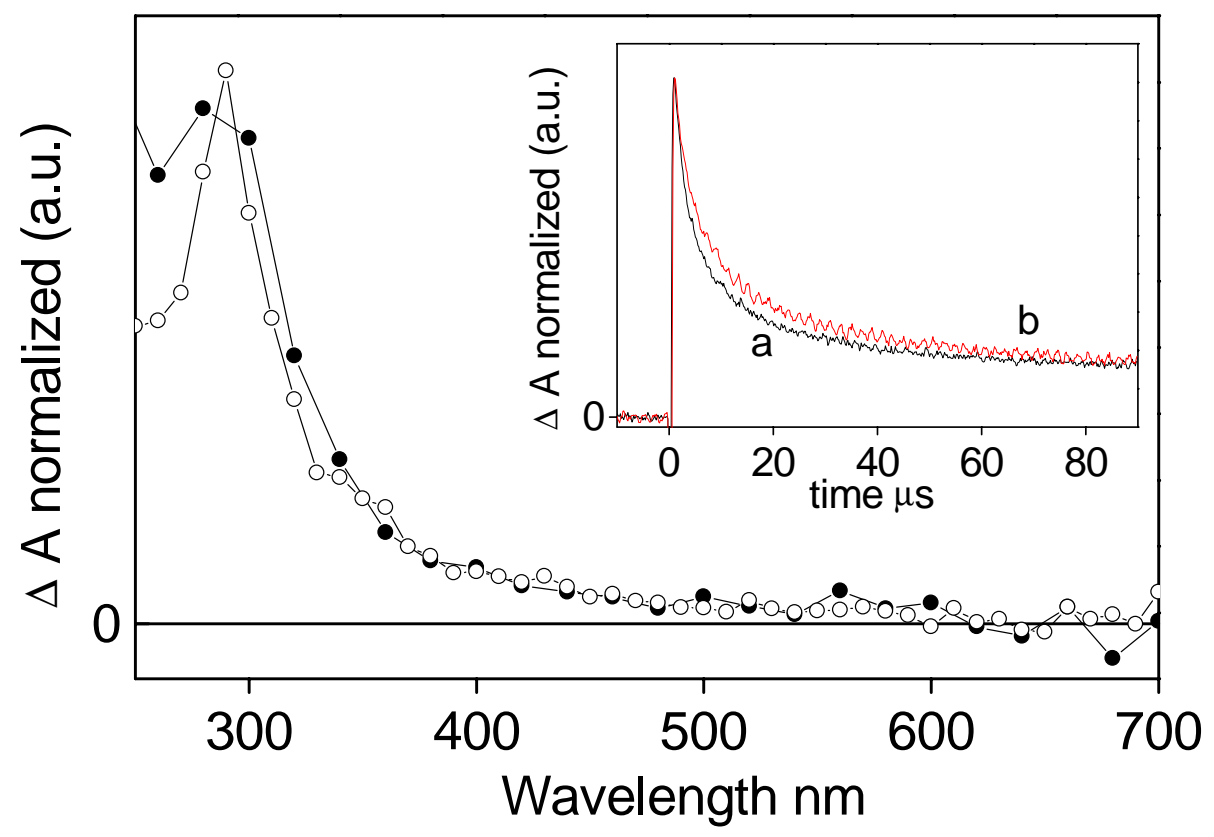

Figure S7. UV-Vis transient absorption spectra recorded $3 \mu$ s after $266 \mathrm{~nm}$ laser excitation of a $\mathrm{N}_{2}$-purged acetonitrile solution of 1,3-diphenylacetone $\left(1.1 \times 10^{-3} \mathrm{M}\right)$ before $(\bullet)$ and after $(\mathrm{O})$ the addition of $\mathrm{Bmim}-\mathrm{Cl}\left(1 \times 10^{-3} \mathrm{M}\right)$. The inset shows the decays monitored at $290 \mathrm{~nm}$ of an acetonitrile solution of 1,3-diphenylacetone before (a) and after (b) the addition of Bmim-Cl. 